\title{
Effect of heat source/sink on MHD free convection flow in a channel filled with nanofluid in the existence of induced magnetic field: an analytic approach
}

\author{
Basant K. Jha ${ }^{1} \cdot$ Gabriel Samaila ${ }^{1}$ (i)
}

Received: 20 February 2020 / Accepted: 25 June 2020 / Published online: 2 July 2020

(c) Springer Nature Switzerland AG 2020

\begin{abstract}
An attempt to examine the relevance of heat source/sink on magnetohydrodynamics free convection flow in a vertical channel with an induced magnetic field is achieved. The analytical solution to the set of the differential equation is obtained by perturbation method for small thermophoresis and Brownian diffusion parameters under the unified thermal boundary condition (isothermal and isoflux boundary condition) for the energy equation. Numerical solution to the flow equation is also obtained by incorporating RKF45 in Maple software. The influence of active parameters such as Hartman number $(\mathrm{Ha})$, magnetic PrandtI number $(\mathrm{Pm})$, heat source/sink parameter $( \pm S)$, Buoyancy ratio $(\mathrm{Br})$, Brownian motion $(\mathrm{Nb})$ and thermophoretic parameter $(\mathrm{Nt})$ on velocity, induced magnetic field, induced current density, nanoparticles concentration, temperature and skin friction are depicted and discussed in detail. Results reveal that the Brownian motion parameter $(\mathrm{Nb})$ and Buoyancy ratio $(\mathrm{Br})$ augment enhances the shear stress whereas the contrast is observed with Hartman number $(\mathrm{Ha})$ and thermophoretic parameter $(\mathrm{Nt})$. Results also reveal that Hartman number $\mathrm{Ha}$ ) and thermophoretic parameter $\mathrm{Nt}$ ) enhances the induced current density while the contrast is true for heat sink parameter $(-S)$. Finally, the temperature of the nanofluid could be enhanced with increase in Brownian motion parameter $(\mathrm{Nb})$ and heat source parameter $(+\mathrm{S})$.
\end{abstract}

Keywords Nanofluid · Isothermal (iso-t) · Isoflux (iso-f) · Heat source/sink · Induced magnetic field

$\begin{array}{ll}\text { Abbreviations } \\ B & \text { Induced magnetic field (Ampere }(A) \mathrm{m}^{-2} \text { ) } \\ \vec{b} & \text { Magnetic field vector (Tesla }(T) \text { ) } \\ T & \text { Temperature of the nannofluid (Kelvin }(\mathrm{K})) \\ \theta & \text { Temperature (dimensionless) } \\ \phi & \text { Concentration (dimensionless) } \\ C & \text { Concentration }\left(\mathrm{mol} \mathrm{dm}^{-3}\right) \\ U & \text { Vertical velocity (dimensionless) } \\ \vec{V} & \text { Velocity vector }\left(\mathrm{ms}^{-1}\right) \\ h & \text { Distance between two vertical walls }(\mathrm{m}) \\ \rho & \text { Fluid density }\left(\mathrm{kgm}^{-3}\right) \\ g & \left.\text { Acceleration due to gravity ( } \mathrm{ms}^{-2}\right) \\ \mathrm{Nb} & \text { Brownian motion parameter (dimensionless) } \\ \beta & \text { Thermal expansion coefficient }\left(\mathrm{K}^{-1}\right)\end{array}$

\section{Abbreviations}

$\vec{B}$

$T$

$\phi$
Ha Hartmann number (dimensionless)

Pm Magnetic Prandtl number (dimensionless)

$S \quad$ Heat source parameter (dimensionless)

$\mu \quad$ Dynamic viscosity of the nanofluid $\left(\mathrm{kg} \mathrm{m}^{-1} \mathrm{~s}^{-1}\right)$

$\mathrm{Nb}$ Brownian motion parameter (dimensionless)

$N t$ Thermophoretic parameter (dimensionless)

$B_{D} \quad$ Brownian diffusion coefficient $\left(m^{2} s^{-1}\right)$

$\sigma \quad$ Electrical conductivity $\left(\mathrm{Sm}^{-1}\right)$

\begin{tabular}{ll}
\multicolumn{2}{l}{ Subscript } \\
$\rho$ & Solid \\
$f$ & Base \\
$c$ & Concentration
\end{tabular}

Gabriel Samaila, gabbbooooo@yahoo.com; Basant K. Jha, basant777@yahoo.co.uk|'Department of Mathematics, Ahmadu Bello University, Zaria, Nigeria. 


\section{Introduction}

The concept of suspension of nanoparticle into a fluid was first introduced by Choi and Eastman [1] to assist in cooling machines that operate at very high temperature. Today, nanofluid is one of the most interesting research area due to its increasing applications in engineering process such as cooling of diverse media, solar collector, thermal absorption, microelectronics, machine processes, nuclear reactor, boiling processes, heat exchanger etc. Nanofluid is a fluid that contains particles between $1-100 \mathrm{~nm}$ in diameter. Ahmad et al. [2] adopted both analytic and numerical method to examine the nanofluid flow physics past a Riga plate. They concluded that the magnitude of the Nusselt number is proportional to the size of the nanoparticles. Sheremet et al. [3] addressed the role of corner heater on MHD nanofluid flow in a porous wavy cavity using a numerical approach. They showed that the magnetic field has a negative impact on the heat transfer. Sheikholeslami and Rokni [4] conducted a study on induced magnetic field impact on MHD between two parallel vertical plates with nanofluid and concluded that the temperature gradient of the nanofluid enhances with suction parameter augment but decreases with thermophoretic parameter augment. Sheikholeslami et al. [5] presented a comprehensive report on nanofluid forced convection flow in porous medium with a magnetic field. They concluded that the heat transfer decreases with Hartmann number. Sheikholeslami [6] numerically examined the transport of MHD nanofluid flow in a permeable medium with radiation effect and concluded that the radiation augment drops the temperature gradient. Vinod and Philip [7] analysed the significant role of the ramp rate on magnetic field in ferrofluid. Their results revealed that the thermal conductivity enhanced with the magnetic field increase.

In the past few decades, the study of magnetohydrodynamic (MHD) has received remarkable consideration owing to its vast area of applications particularly in: petroleum, chemical technology, engineering, geophysics and environmental sciences. In most of the study on hydromagnetic natural convection flow, the impact of the induced magnetic field has been ignored in order to simplify the mathematical formulation. The induced magnetic field has the potential to creates its own magnetic field which consequently modifies the real magnetic field. Thus, the inclusion of the impact of the induced magnetic in the governing equation becomes a necessity when formulating hydromagnetic related problems. Early investigations such as [8-17] have analysed the impact of the induced magnetic field on MHD.
They demonstrated that the velocity of the fluid flow always decreases with induced magnetic field strength. Singh and Sarveshanand [18] also examined the effect of the induced magnetic field on MHD free convection in a vertical channel. Their results suggested that the magnetic field enhanced with suction injection augment but decreases induced current density and velocity profile. Multi-physics simulation of dendritic grows in Lorentz force assisted solidification has been addressed by Cao et al. [19]. Feng et al. [20] analysed the role horizontal, rotational and vertical magnetic field on convection in a magnetically levitated droplet. Their results suggested that the magnetic field plays a pivotal role in thermal conductivity measurement. They also stressed that the magnetic field tend to induce forced convection along the azimuthal direction. Mehryan et al. [21] recent presented melting characteristic of phase change materials under the influence of non-uniform magnetic field resulted by magnetic source.

The study on the implication of heat source/sink on MHD flows continues to inspire researchers in various fields owing to its applications in industries. The role of heat source in a fluid transport is to increase its thermal conductivity which consequently increases the fluid temperature whereas heat sink, on the other hand, decreases the thermal conductivity which consequently decreases the temperature of the fluid. Bhattacharya [22] analysed the combined impact of heat source/sink, suction/injection and radiation on the boundary layer past a shrinking surface. They concluded that the thermal boundary layer thickness has a decreasing tendency with Prandtl number, heat sink and radiation parameters variation but enhanced with heat source parameter. Kumar and Singh [23] investigated the importance of heat source/sink on free convective flow. The outcome of their study indicated that the velocity increased with heat source augment but they stressed that the opposite is true for the heat sink. Alsabery et al. [24] scrutinized the significant role of heat source/sink on natural convective flow in a cylindrical cavity. Their result revealed that the nanoparticles volume fraction augment increases heat transfer. Tlau and Ontela [25] examined the importance of heat source/sink on MHD nanofluid using homotopy analysis. The concluded that the Nusselt number and skin friction is higher in heat generation compared the heat absorption. Chu et al. [26] performed a CFD simulation on the performance of the heat sink for diminishing junction temperature. Wang et al. [27] numerically scrutinised the role of thermal and hydraulic behaviour of micro latticed pin fin in the heat sink. They stressed that the latticed pin has the potential to decrease the intensity of the heat sink when considering the weight reduction. Roy et al. [28] addressed the importance of trapezoidal heat source on a free convection flow fixed on a 
cylindrical cavity. They illustrated that the magnitude of the streamline is proportional to the strength of the heat source. Considering the industrial importance of heat generation/absorption (heat source/sink) in the present days, it is the interest of this paper to study the implication of heat source/sink on the MHD in the presence of the induced magnetic field.

To the best of the authors' knowledge, the effect of heat source/sink on MHD using the mixed boundary condition has not been considered. Thus, this paper aims to study the implication of heat generation/absorption on MHD convective flow of viscous fluid in a vertical channel filled with nanoparticles in the existence of induced magnetic field. The paper is closely related to the work of Sheikholeslami and Rokni [4]. The governing flow equations are remodelled in the absence of suction/injection to include the impact of heat generation/absorption in the energy equation. It is imperative to remark that due to the coupled nature of the flow equations, Sheikholeslami and Rokni [4] presented only the numerical approach to the problem using isoflux boundary condition. The novelty of this article over Sheikholeslami and Rokni [4] is (a) The inclusion of the heat source/sink in the energy equation (b) the existence of the analytical solution to the governing equation by perturbation method under the isothermal, isoflux thermal boundary conditions (c) The ability to obtain the exact solution to governing equations using isothermal boundary condition in the absence of heat source/sink. The influence of the prominent parameters on the velocity, temperature, induced magnetic field, current density and the rate of nanoparticles concentration have been depicted using graphs.

\section{Mathematical analysis}

In this paper, nanofluid flow through two infinite vertical walls in the existence of the induced magnetic field is considered. The distance between the two vertical walls is considered as $h$. The $x$-axis is considered to be vertically upward along the wall and the $y$-axis is considered perpendicular to the vertical wall as depicted in Fig. 1. Uniform magnetic field $b_{0}$ is applied perpendicular to the two vertical walls. The walls at $y=0$ and $y=h$ are respectively considered to be electrically non - conducting and conducting. The two plates are assumed to be at constant temperature (isothermal case) or constant heat flux (isoflux case). The variable describing the fluid flow only depend on y-coordinate, because the plates are of infinite extent axis. For a fluid with electrical conductivity $(\sigma)$, we denote the induced magnetic

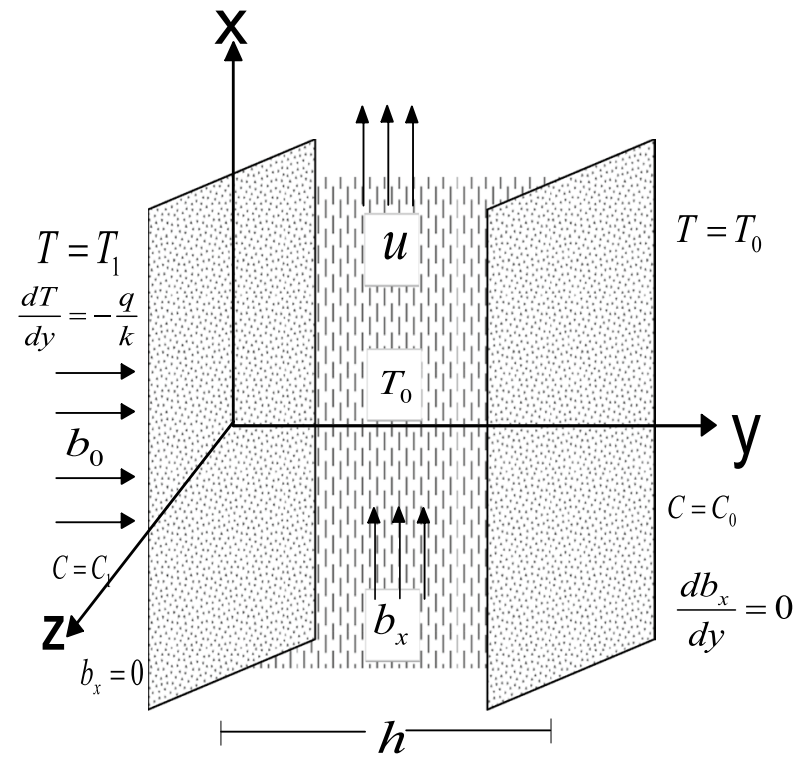

Fig. 1 Physical model

field along the $x$-axis as $b_{x}$. The magnetic field and the velocity vectors considered are $\vec{b}=\left[b_{x}, b_{0}, 0\right]$ and $\vec{v}=\left[v, u_{0}, 0\right]$ respectively.

The Maxwell equations utilised in formulating the flow equation are:

$\vec{\nabla} \times \vec{E}=-\frac{\partial \vec{B}}{\partial t} \quad$ Faraday's law

$\vec{\nabla} \cdot \vec{B}=0$ Solenoidal consevation law

$\vec{\nabla} \times \vec{B}=\mu_{e} \vec{\jmath} \quad$ Ampare's circuital law

$\vec{F}=\vec{J} \times \vec{B} \quad$ Lorentz law

Accordingly, the magnetic induction equation utilised is written as:

$\nabla^{2} \vec{B}=\sigma \mu_{e} \vec{\nabla} \times(\vec{v} \times \vec{B})$.

Using the above assumption, the governing equation describing the fluid transport can be written following Sarveshanand and Singh [18] and Sheikholeslami and Rokni [4] as

$\frac{\mu}{\rho f} \frac{d^{2} u}{d y^{2}}+\frac{\mu_{e} b_{0}}{\rho f} \frac{d b_{x}}{d y}+g \beta\left(T-T_{0}\right)+g \beta_{c}\left(C-C_{0}\right)=0$,

$\frac{1}{\mu_{e} \sigma} \frac{d^{2} b_{x}}{d y^{2}}+b_{0} \frac{d u}{d y}=0$ 
$\alpha \frac{d^{2} T}{d y^{2}}+\frac{\rho C_{p}}{\rho C_{f}}\left[D_{B}\left(\frac{d C}{d y} \frac{d T}{d y}\right)+\frac{D_{T}}{T_{0}}\left(\frac{d T}{d y}\right)^{2}\right]+S^{\prime}=0$,

$D_{B} \frac{d^{2} T}{d y^{2}}+\frac{D_{T}}{T_{0}} \frac{d^{2} C}{d y^{2}}=0$

With boundary conditions:

$C=C_{0}, u=0, b_{x}=0, T=T_{0}+\frac{d q}{k}$ or $\frac{d T}{d y}=-\frac{q}{k}$, at $\quad y=0$,

$C=C_{0}, u=0, \frac{d b x}{d y}=0, T=T_{0}$, at $y=1$,

We defined the dimensionless parameters and variables following Sarveshanand and Singh [18] and Sheikholeslami and Rokni [4] as

$Y=\frac{y}{h}, U=\frac{\mu u}{\rho f g \beta h^{2} \Delta T}, B=\sqrt{\frac{\mu_{e} b_{x}}{\rho f} \frac{\mu}{\beta h^{2} \rho f g \Delta T}}$

$\theta=\Delta T^{-1}\left[T-T_{0}\right], \quad \phi=\frac{C-C_{0}}{\Delta C}, \Delta C=C-C_{0}$

$P m=\frac{\mu}{\rho f \alpha} \sigma \mu_{e}, H a=\frac{B_{0} h}{v} \sqrt{\frac{\mu_{e}}{\rho}}, S=\frac{S^{\prime} h^{2}}{\alpha \Delta T}$

$B r=\frac{\beta_{c} \Delta C}{\beta \Delta T}, N b=\frac{\left(\rho C_{p}\right)_{p} D_{B} \Delta C}{\left(\rho C_{p}\right)_{f} \alpha}, N t=\frac{\left(\rho C_{p}\right)_{p} D_{B} \Delta T}{\left(\rho C_{p}\right)_{f} \alpha T_{0}}$.

Where $(\Delta T)$ is $\left(T_{1}-T_{0}\right)$ or $q h / k$ accordingly as left wall is maintained at constant temperature $T_{1}$ or constant heat flux $q$. The final dimensionless equations are:

$\frac{d^{2} U}{d Y^{2}}+H a \frac{d B}{d Y}+\theta+B r \phi=0$,

$\frac{d^{2} B}{d Y^{2}}+H a P m \frac{d U}{d Y}=0$

$\frac{d^{2} \theta}{d Y^{2}}+N t\left(\frac{d \theta}{d Y}\right)^{2}+N b \frac{d \theta}{d Y} \frac{d \phi}{d Y}+S=0$

$\frac{d^{2} \phi}{d Y^{2}}+\frac{N t}{N b} \frac{d^{2} \theta}{d Y^{2}}=0$

With boundary conditions: $\phi=1, \quad B=0, \quad U=0, \quad A^{*} \frac{d \theta}{d Y}+B^{*} \theta=C^{*}, \quad$ at $Y=0$,

$\phi=0, \frac{d B}{d Y}=0, U=0, \quad \theta=0, \quad$ at $Y=1$.

where $A^{*}, B^{*}$ and $C^{*}$ are constants such that with: $A^{*}=0, B^{*}=1$ and $C^{*}=1$ denotes Isothermal heating condition (Iso-T) and. $A^{*}=1, B^{*}=0$ and $C^{*}=-1$ denotes Isoflux heating condition (Iso-F) respectively.

The skin friction and induced density are defined as $C_{f}=U^{\prime}(0)$ and $J=-\frac{d B}{d Y}$ respectively.

\subsection{Perturbation method}

Considering that Eqs. (12) and (13) are independent of Eqs. (10) and (11). Therefore, Eq. (10) and (11) can be solved after developing the solution for $\phi$ and $\theta$ profiles. Ahmad, Asghar, and Afzal [2] reported that the magnitude of the thermophoretic and Brownian diffusion is very small. Therefore consider $N b$ and $N t$ of $O(\varepsilon)$, as $\varepsilon \rightarrow 0$ and expand $\theta$ and $\phi$ in small parameter $\varepsilon$ such that;

$\theta=\theta_{0}+\varepsilon \theta_{1}+\ldots$

$\phi=\phi_{0}+\varepsilon \phi_{1}+\ldots$

The $\varepsilon^{0}$ and $\varepsilon^{1}$ boundary conditions for Eqs. (10)-(13) are $\phi=1, \quad B=0, \quad U=0, \quad A^{*} \frac{d \theta}{d Y}+B^{*} \theta=C^{*}, \quad$ at $Y=0$, $\phi=0, \frac{d B}{d Y}=0, U=0, \quad \theta=0, \quad$ at $Y=1$.

and

$\phi=0, \quad B=0, \quad U=0, \quad A^{*} \frac{d \theta}{d Y}+B^{*} \theta=0, \quad$ at $Y=0$,

$\phi=0, \frac{d B}{d Y}=0, U=0, \quad \theta=0, \quad$ at $Y=1$.

respectively. Where, Isothermal (Iso-T): $A^{*}=0, B^{*}=1, C^{*}=1$, Isoflux (Iso-F): $A^{*}=1, B^{*}=0, C^{*}=-1$.

The analytic solution of the above boundary value problem is given as

$\theta(Y)=H_{106}+H_{107} Y+H_{108} Y^{2}+H_{109} Y^{3}$,

$\phi(Y)=H_{110}+H_{111} Y+H_{112} Y^{2}+H_{113} Y^{3}$, 
$B(Y)=H_{114}+H_{115} Y+H_{116} Y^{2}+H_{117} Y^{3}+H_{118} Y^{4}+H_{119} \exp \left(H_{14} Y\right)+H_{120} \exp \left(H_{15} Y\right)$,

$U(Y)=H_{121}+H_{122} Y+H_{123} Y^{2}+H_{124} Y^{3}+H_{125} \exp \left(H_{14} Y\right)+H_{126} \exp \left(H_{15} Y\right)$

$U^{\prime}(Y)=H_{122}+2 H_{123} Y+3 H_{124} Y^{2}+H_{14} H_{125} \exp \left(H_{14} Y\right)+H_{15} H_{126} \exp \left(H_{15} Y\right)$

where $H_{i}, i=1,2, \ldots 126$ are constants.

\subsection{Exact solution}

The exact solution for Eqs. (10)-(13) are obtained using isothermal boundary condition and it can be expressed as:

$$
\begin{aligned}
\theta(Y)= & P_{3}+P_{2} \exp \left(m_{1} Y\right) \\
\phi(Y)= & P_{5}+P_{6} Y+\frac{P_{4} \exp \left(m_{1} Y\right)}{m_{1}^{2}} \\
B(Y)= & P_{53}+P_{14} Y+P_{16} Y^{2}+P_{17} \exp \left(m_{1} Y\right) \\
& +P_{51} \exp \left(m_{2} Y\right)+P_{52} \exp \left(m_{3} Y\right) \\
U(Y)= & P_{54}+P_{55} Y+P_{56} \exp \left(m_{1} Y\right)+P_{57} \exp \left(m_{2} Y\right)+P_{58} \exp \left(m_{3} Y\right) \\
U^{\prime}(Y)= & P_{55}+m_{1} P_{56} \exp \left(m_{1} Y\right)+m_{2} P_{57} \exp \left(m_{2} Y\right) \\
& +m_{3} P_{58} \exp \left(m_{3} Y\right)
\end{aligned}
$$

where $C_{3}=P_{47}, C_{4}=P_{50}$ and $P_{i}, i=1,2,3, \ldots 58$ are constants defined in "Appendix A".

\section{Results and discussion}

The influence of heat source/sink on magnetohydrodynamics (MHD) free convection in a vertical channel filled with nanofluid is examined. The solution to the sets of

Table 1 Variation of skin friction for isothermal boundary condition with $\mathrm{Pm}(\mathrm{Ha}=10, \mathrm{Br}=1, \mathrm{Nb}=0.1, \mathrm{Nt}=0.1$,

\begin{tabular}{llll}
\hline Pm & $\begin{array}{l}\text { Exact solution } \\
S=0\end{array}$ & $\begin{array}{l}\text { Analytic solution } \\
S \rightarrow 0\end{array}$ & $\begin{array}{l}\text { Numerical solution } \\
S=0\end{array}$ \\
\hline 0.1 & 0.434726 & 0.434726 & 0.434726 \\
0.2 & 0.347330 & 0.347330 & 0.347330 \\
0.6 & 0.242843 & 0.242843 & 0.242843 \\
0.6 & 0.224866 & 0.224866 & 0.224866 \\
0.7 & 0.210474 & 0.210474 & 0.210474 \\
0.8 & 0.198607 & 0.198607 & 0.198607 \\
0.9 & 0.188596 & 0.188596 & 0.188596 \\
1.0 & 0.180000 & 0.180000 & 0.180000 \\
\hline
\end{tabular}

differential equations is obtained by perturbation method using unified thermal boundary condition. The results obtained using perturbation method is validated through comparison with exact and numerical solutions for isothermal boundary condition only. The numerical solution is obtained using RKF45 in Maple software while the exact solution is obtained through the method of undetermined coefficient. Table 1 demonstrates the accuracy of the present approach. The influence of Hartmann number $(\mathrm{Ha})$, heat source/sink parameter $( \pm S)$, Buoyancy ratio $(\mathrm{Br})$, magnetic Prandtl number $(\mathrm{Pm})$, Brownian motion parameter $(\mathrm{Nb})$, and thermophoretic parameter $(\mathrm{Nt})$ on the velocity $(V)$, induced magnetic field $(B)$, induced current density $(J)$, nanoparticles concentration $(\phi)$, temperature distribution $(\theta)$ and skin friction under the unified thermal boundary condition are presented. To accurately examine the role of the active parameters on the fluid flow, the values of $\mathrm{Ha}, \mathrm{Pm}, \mathrm{Br}, \mathrm{Nb}, \mathrm{Nt}$ and $\mathrm{S}$ are chosen as: $5 \leq H a \leq 15,0.4 \leq P m \leq 1.20 .5 \leq B r \leq 2.0,0.01 \leq N b \leq 0.4$, $0.01 \leq N t \leq 0.4$.

\subsection{Influence of the prominent parameters}

Figures 2, 3, 4 depict the effects of varying the Hartmann number $(\mathrm{Ha})$, magnetic Prandtl number $(\mathrm{Pm})$ and Buoyancy ratio $(B r)$ on the velocity $(V)$, induced magnetic field $(B)$ and induced current density $(J)$ under isothermal and isoflux thermal boundary conditions. It is evident from Fig. 2a, c that the velocity and induced current density profile of the fluid retards with enhancement in Hartmann number $(\mathrm{Ha})$. The velocity profile experienced sudden reduction owing to the increase in the Lorentz force which opposes the flow. Regarding the induced magnetic field, an increase in the values of Hartmann number $(\mathrm{Ha})$ has an increasing tendency on the induced magnetic field as shown in Fig. 2b. It can be seen from Fig. 3a, b that in the existence of heat generation/absorption, both velocity and induced magnetic field profile reveals to be inversely proportional to increase in magnetic Prandtl number $(P m)$. Thus, a rise in the values of magnetic Prandtl number $(\mathrm{Pm})$ induces a destabilizing influence of flow formation within the channel. The contrast result is observed on induced 


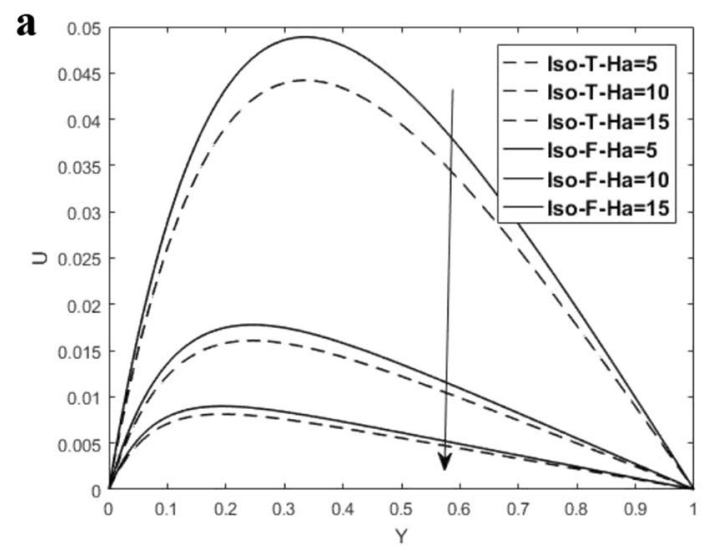

b
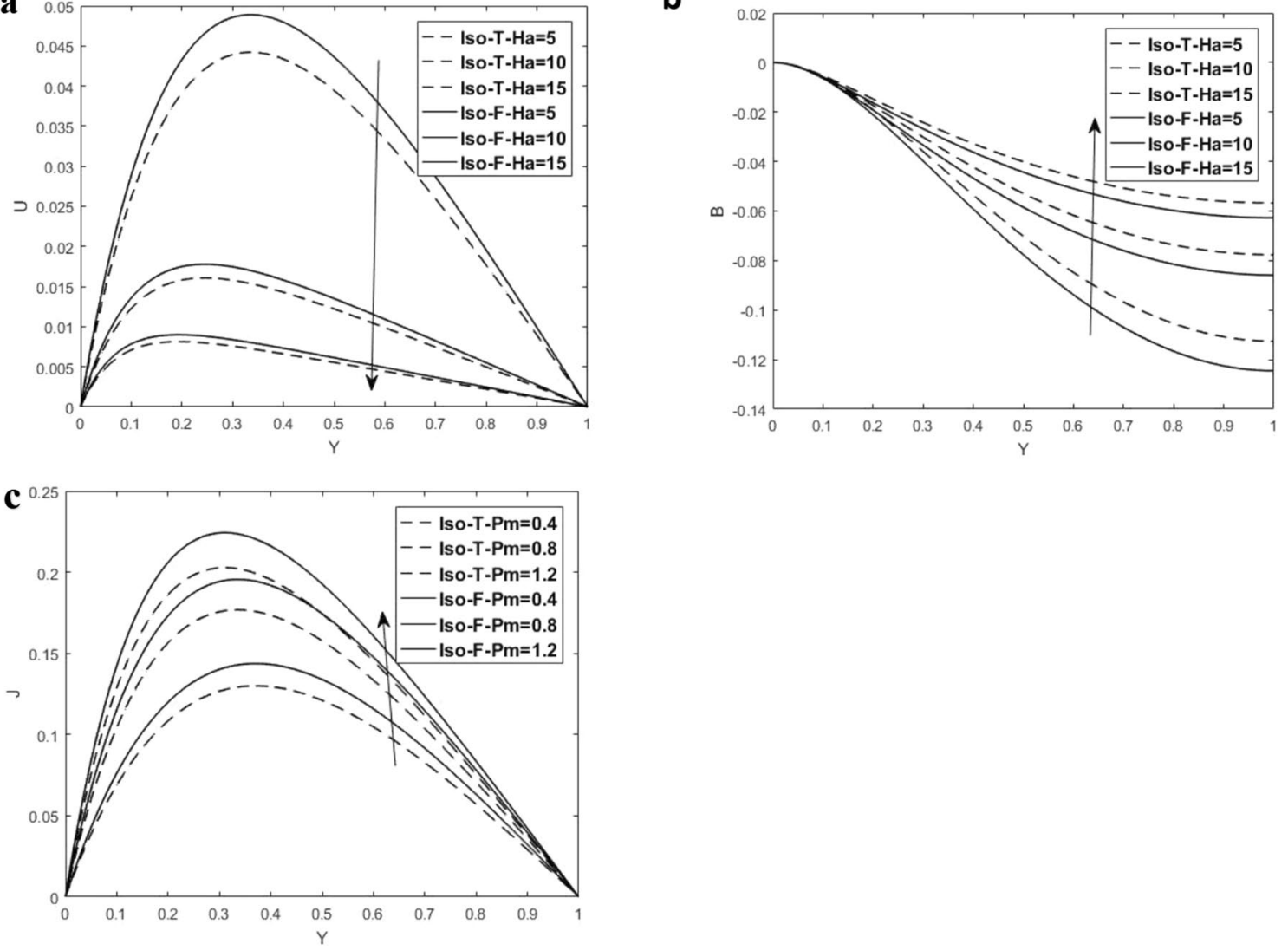

Fig. 2 Variation of $\mathrm{Ha}$ on a Velocity $\mathbf{b}$ Induced magnetic field and $\mathbf{c}$ Induced current density for $\mathrm{Nb}=0.1, \mathrm{Nt}=0.1, \mathrm{~S}=0.2, \mathrm{Pm}=0.8, \mathrm{Br}=1$

current density, as $P m$ is observed to support induced current density as shown in Fig. 3 c. From Fig. $4 a-c$ it is obvious that in the existence of magnetic field and heat generation/absorption, velocity and induced current density profile could be enhanced by increasing the Buoyancy ratio $(\mathrm{Br})$. A close inspection of the Fig. 4 a also reveals that velocity fluid is at its peak under isoflux heating situations in comparison to isothermal heating. This phenomena is true and can be observed for all values of Buoyancy ratio. However, it is interesting to observe that, Buoyancy ratio $(\mathrm{Br})$ variation is observed to have a decreasing effect on the induced magnetic field as portrayed in Fig. $4 \mathrm{~b}$.

Figures 5, 6, 7, 8 demonstrate the influence of Brownian motion parameter $(\mathrm{Nb})$, thermophoretic parameter $(\mathrm{Nt})$, heat source parameter $(S)$ and heat sink parameter $(-S)$ variation on the velocity $(V)$, induced magnetic field $(B)$, induced current density $(J)$, nanoparticles concentration $(\phi)$ and temperature distribution $(\theta)$ for isothermal and isoflux thermal boundary conditions. Observation from
Fig. 5a-e shows that the velocity, induced current density, temperature and the rate of nanoparticles concentration enhances with Brownian motion parameter $(\mathrm{Nb})$ augment whereas contrary behaviour is noticed for induced magnetic field. It is obvious from Fig. 6a, c-e that the thermophoretic parameter $(\mathrm{Nt})$ augment leads to the decrease in the velocity and induced current density profile, temperature distribution and rate of nanoparticles concentration of the nanofluid but contrary trend is observed in the case of the induced magnetic field as portrayed in Fig. $6 \mathrm{~b}$.

The presence of the heat source parameter $(S)$ in the temperature field has several engineering importance. It is noticed from Fig. 7a, c, d that heat generation supports velocity, induced current density and the temperature distribution with an observable increase in flow formation whereas it destabilizes the induced current density and the rate of nanoparticles concentration. The fact behind this phenomena is that with growing the heat generation parameter, the fluid becomes warmer thereby adding 


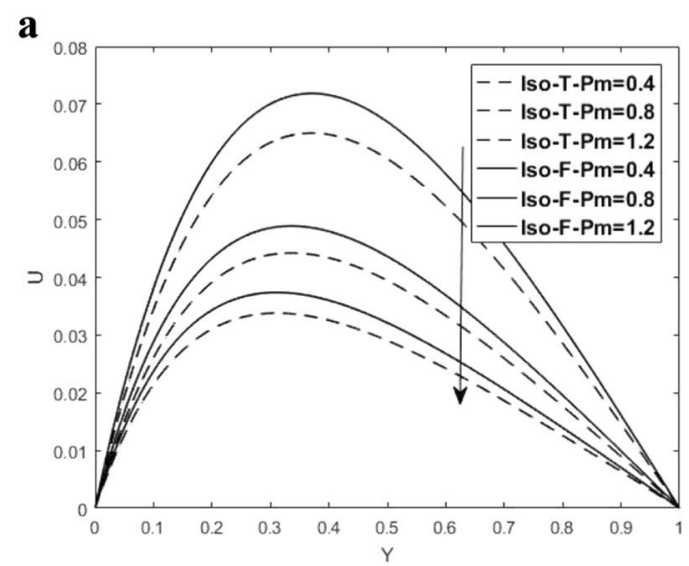

b
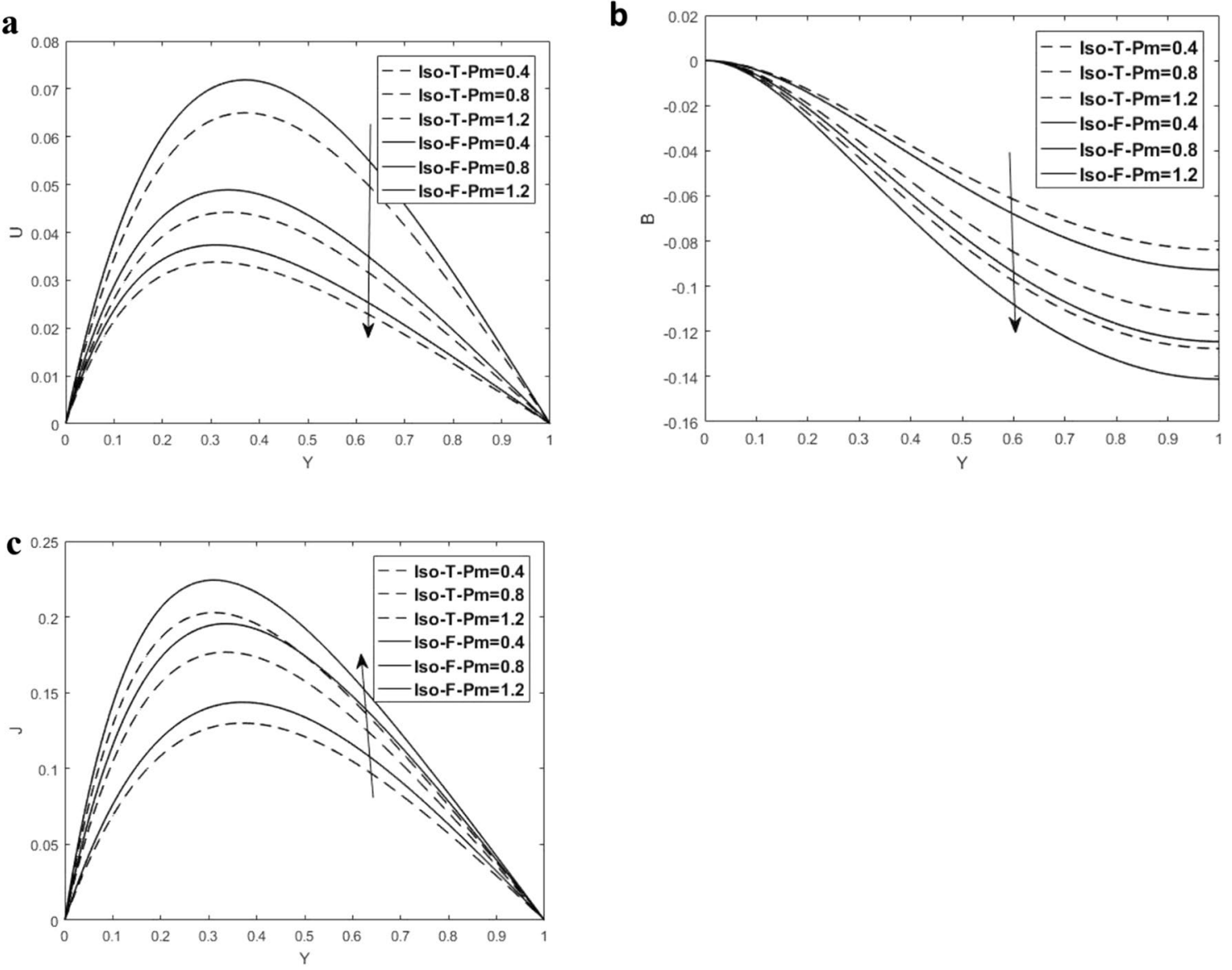

Fig. 3 Variation of $P m$ on $\mathbf{a}$ Velocity $\mathbf{b}$ Induced magnetic field and $\mathbf{c}$ Induced current density for $\mathrm{Nb}=0.1, \mathrm{Ha}=5, \mathrm{~S}=0.2, \mathrm{Nt}=0.1, \mathrm{Br}=1$

to the temperature field and consequently increasing the velocity and induced current density of the fluid. It is interesting to observe from Fig. 7a-e that there is significant variation in the velocity, induced magnetic field, induced current density, rate of nanoparticles concentration and temperature distribution when the isoflux boundary condition is applied whereas there is almost insignificant influence under isothermal heating. The reason behind this phenomenon is that with isoflux heating, increase in heat generations reduces the rate of heat transfer thereby enhancing the temperature towards the left wall with constant heat flux whereas the influence of heat generation/absorption becomes almost insignificant under the isothermal heating since the temperature of the walls is not changing. It is clear from the Fig. $8 a-e$ that increase in heat absorption parameter $(-S)$ destabilizes flow formation with an observable reduction in velocity, induced current density profile and temperature distribution whereas it supports the induced magnetic field and the rate of nanoparticles concentration.

Table 1 shows the comparison of the results obtained using the perturbation method, exact solution and the numerical solution for various values of $P m$ on skin friction under isoflux thermal boundary condition. It is obvious that the skin friction decreases with magnetic Prandtl number augment. Table 2 demonstrates the impact of the heat source/sink parameter variation on skin friction for isoflux boundary condition. It is worthy to remark that the skin friction enhanced with heat source parameter augment but decreases with heat sink parameter augment. 


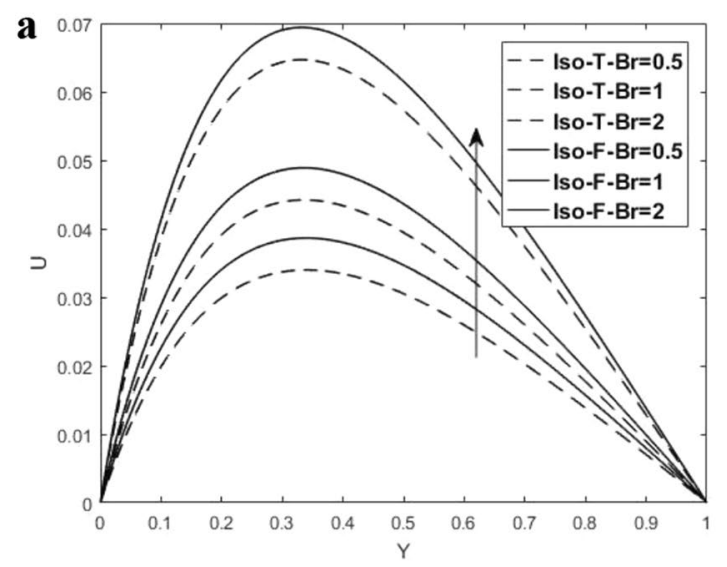

b
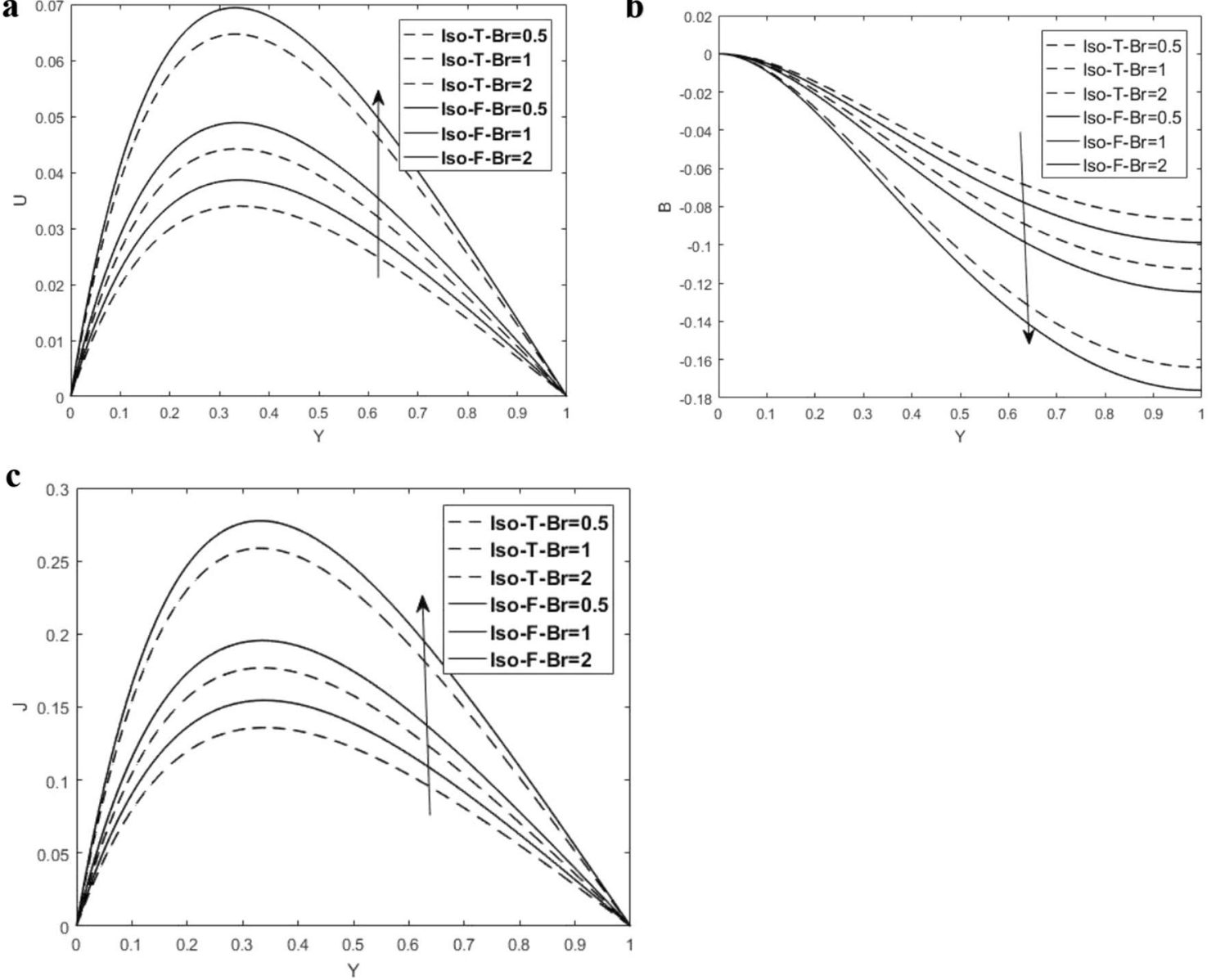

Fig. 4 Variation of $\mathrm{Br}$ on $\mathbf{a}$ Velocity $\mathbf{b}$ Induced magnetic field and $\mathbf{c}$ Induced current density for $\mathrm{Nb}=0.1, \mathrm{Ha}=5, \mathrm{~S}=0.2, \mathrm{Pm}=0.8, \mathrm{Nt}=0.1$

\section{Conclusion}

Magnetohydrodynamic convective flow of viscous fluid in a vertical channel filled with nanofluid under the unified thermal boundary condition has been presented. The influence of induced magnetic field and heat source/sink are taken into consideration. The Perturbation method is adopted to solve the governing equations, under isothermal and isoflux boundary conditions. Effects of $\mathrm{Ha}, \mathrm{Pm}, \pm S$, $\mathrm{Br}, \mathrm{Nb}$ and $\mathrm{Nt}$ on velocity, induced magnetic field, induced current density, nanoparticles concentration, temperature distribution and skin friction for the two thermal boundary conditions are fully presented. The results indicated that: (i) The shear stress enhances with growing $N b, \mathrm{~S}$ and $\mathrm{Br}$ whereas it reduces with other active parameters.

(ii) The strength of the induced magnetic field enhances with increasing values of $\mathrm{Ha}, \mathrm{Nt}$ and $-\mathrm{S}$ whereas it decreases with increasing values of the other active parameters.

(iii) The induced current density enhances with increasing values of $\mathrm{Pm}, \mathrm{Br}, \mathrm{Nb}$, and heat source parameter(S) whereas it decreases with other active parameters augment. 

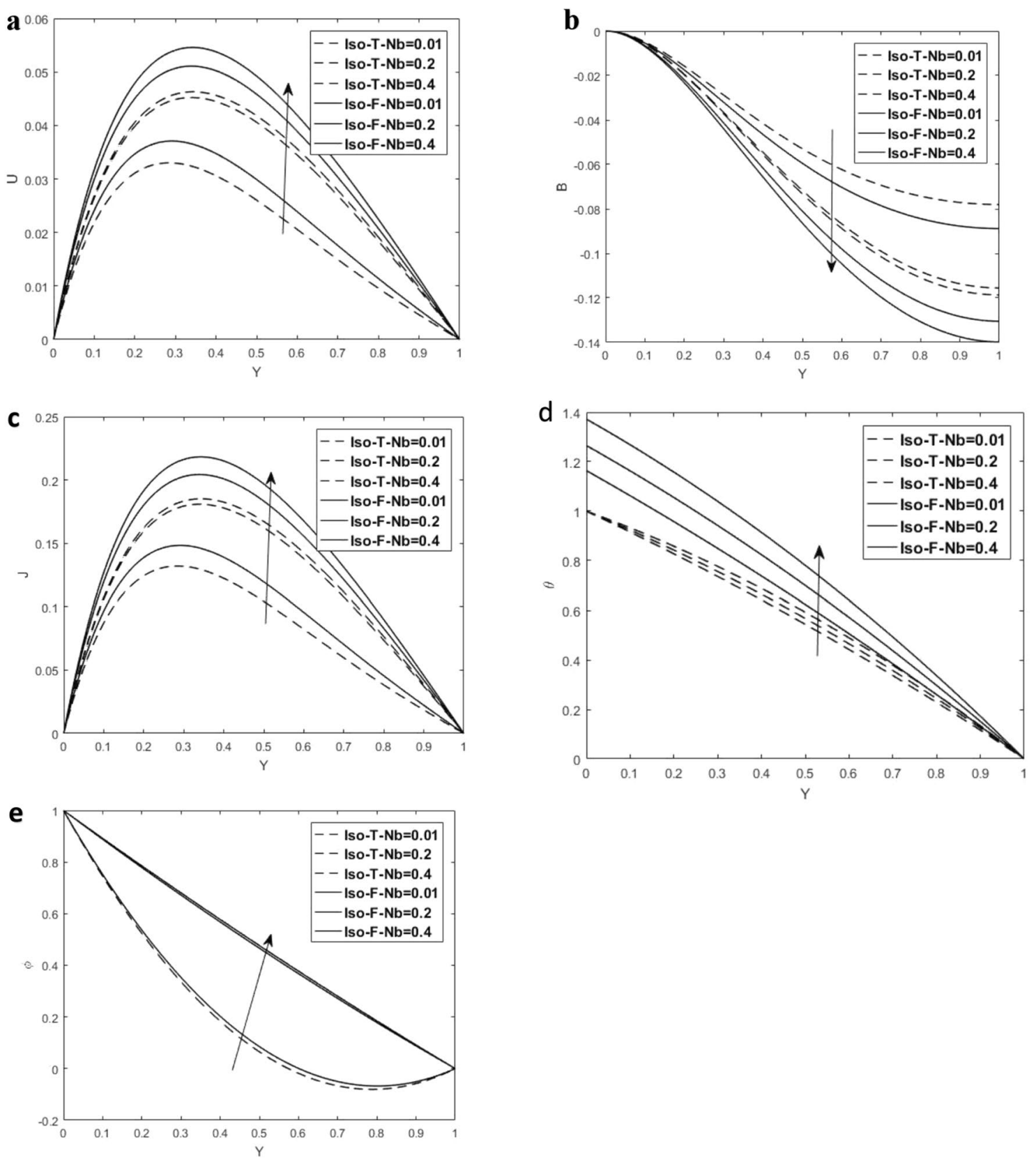

Fig. 5 Variation of $\mathrm{Nb}$ on a Velocity $\mathbf{b}$ Induced magnetic field $\mathbf{c}$ Induced current density $\mathbf{d}$ Temperature and $\mathbf{e}$ Concentration for $\mathrm{Nt}=0.1, \mathrm{Ha}=5, \mathrm{~S}=0.2, \mathrm{Pm}=0.8, \mathrm{Br}=1$

(iv) The temperature distribution of the nanofluid enhances with increasing values of $\mathrm{Nb}$ and $\mathrm{S}$ but decrease with $N t$ and $-S$ augment.

(v) The nanoparticles diffusion enhances with increasing values of $\mathrm{Nb}$ and $-\mathrm{S}$ whereas it decreases with $N t$ and $S$ augment. (vi) The skin friction enhances with increasing values of $S$ whereas it decreases with $-S$ augment. 
a

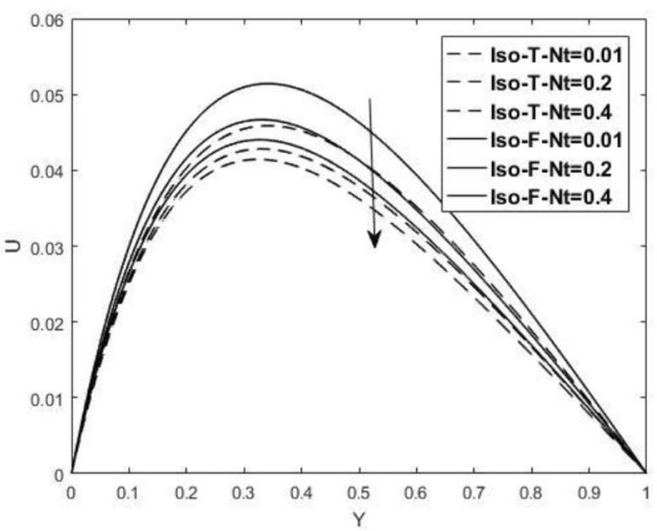

c

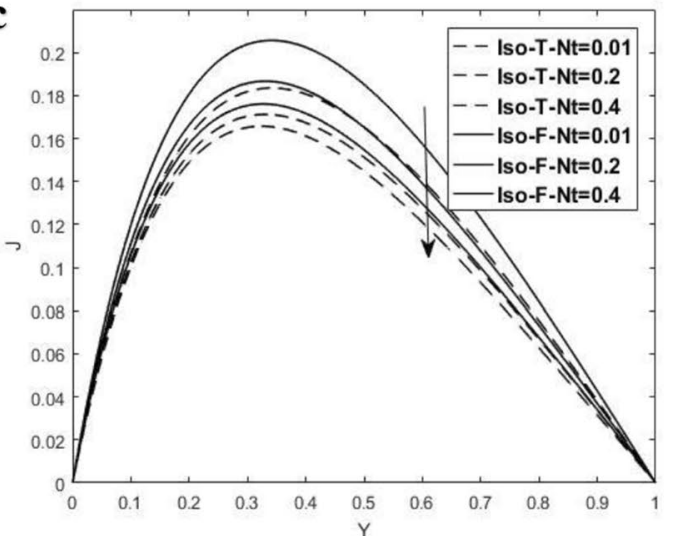

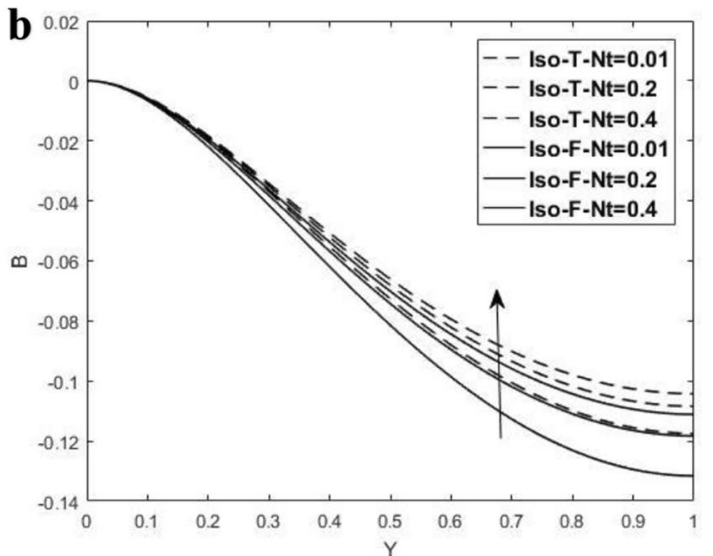

d

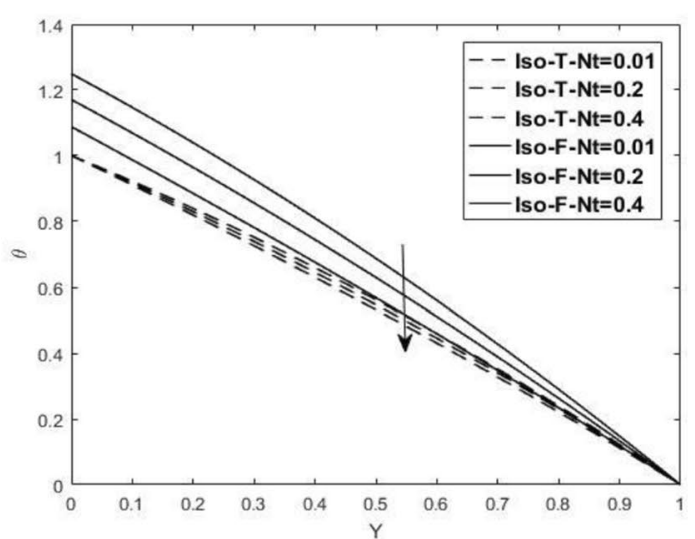

$\mathrm{e}$

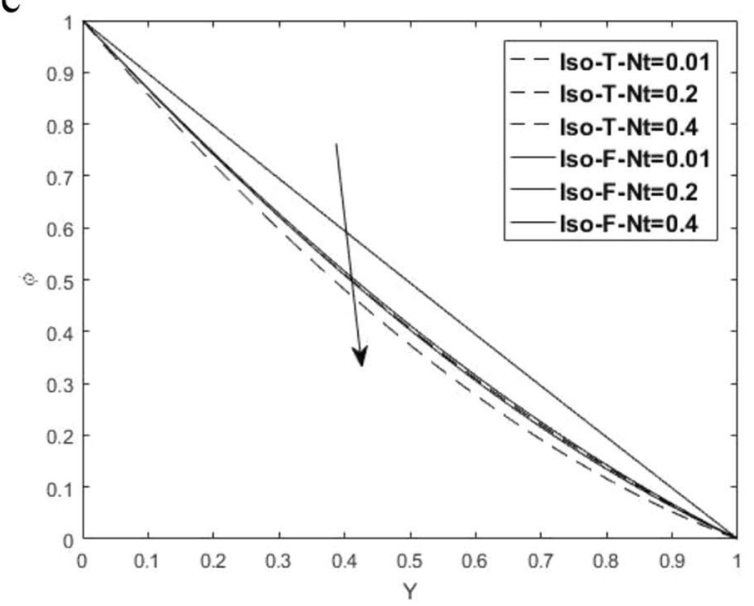

Fig. 6 Variation of $N t$ on a Velocity $\mathbf{b}$ Induced magnetic field $\mathbf{c}$ Induced current density $\mathbf{d}$ Temperature and $\mathbf{e}$ Concentration for $\mathrm{Nb}=0.1, \mathrm{Ha}=5, \mathrm{~S}=0.2, \mathrm{Pm}=0.8, \mathrm{Br}=1$ 
a

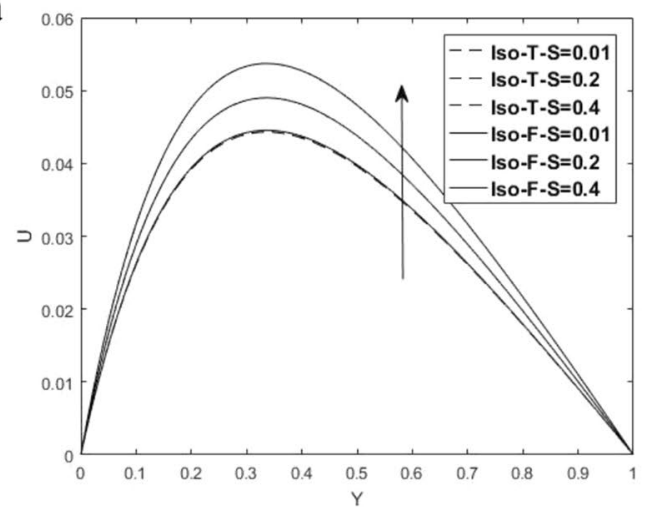

C

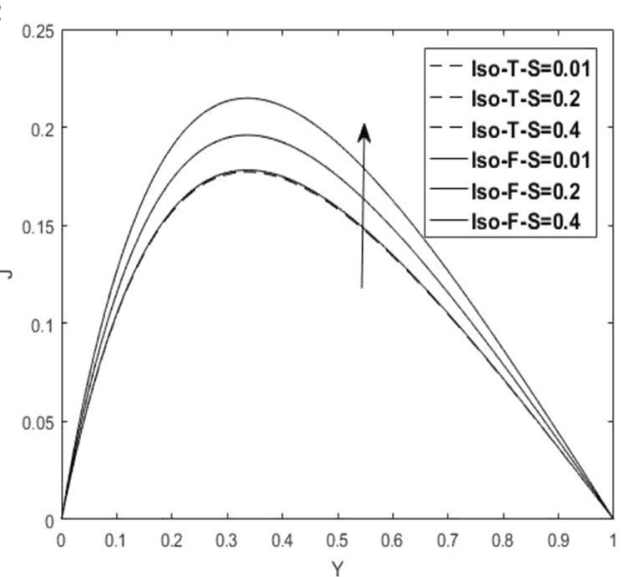

b

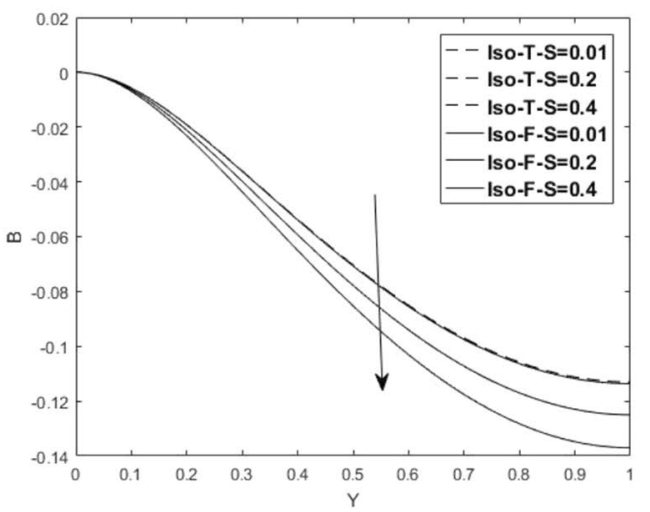

d

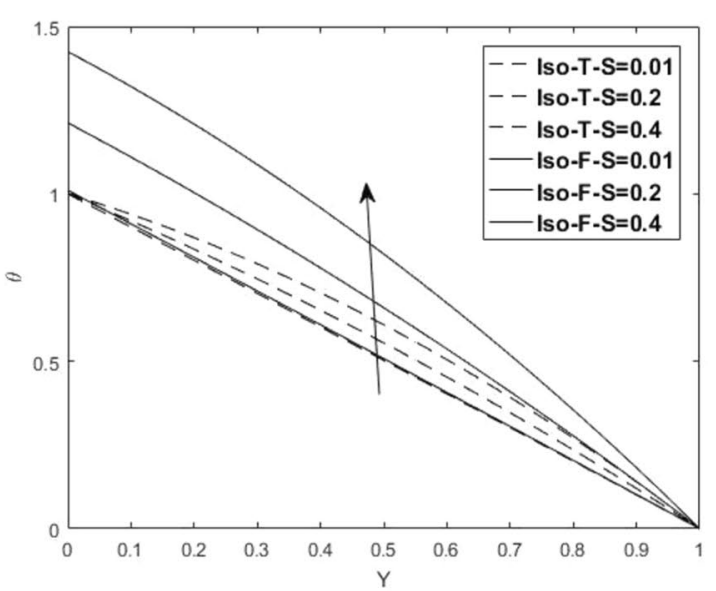

e

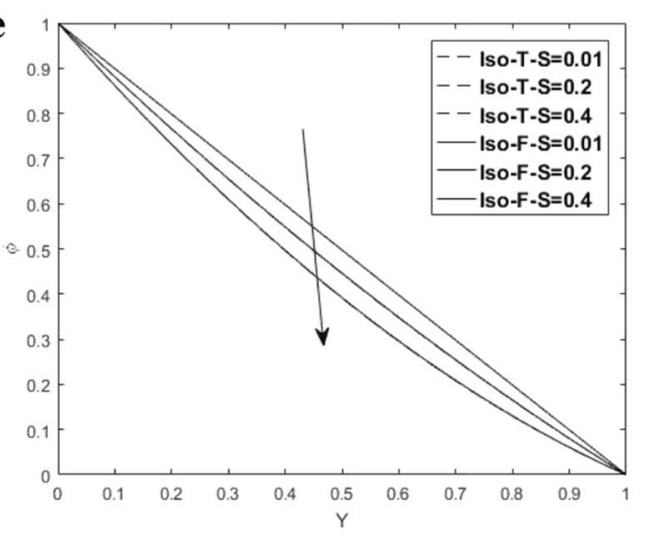

Fig. 7 Variation of $S$ on a Velocity b Induced magnetic field c Induced current density d Temperature and e Concentration for $\mathrm{Nb}=0.1, \mathrm{Ha}=5, \mathrm{~S}=0.2, \mathrm{Pm}=0.8, \mathrm{Br}=1$ 

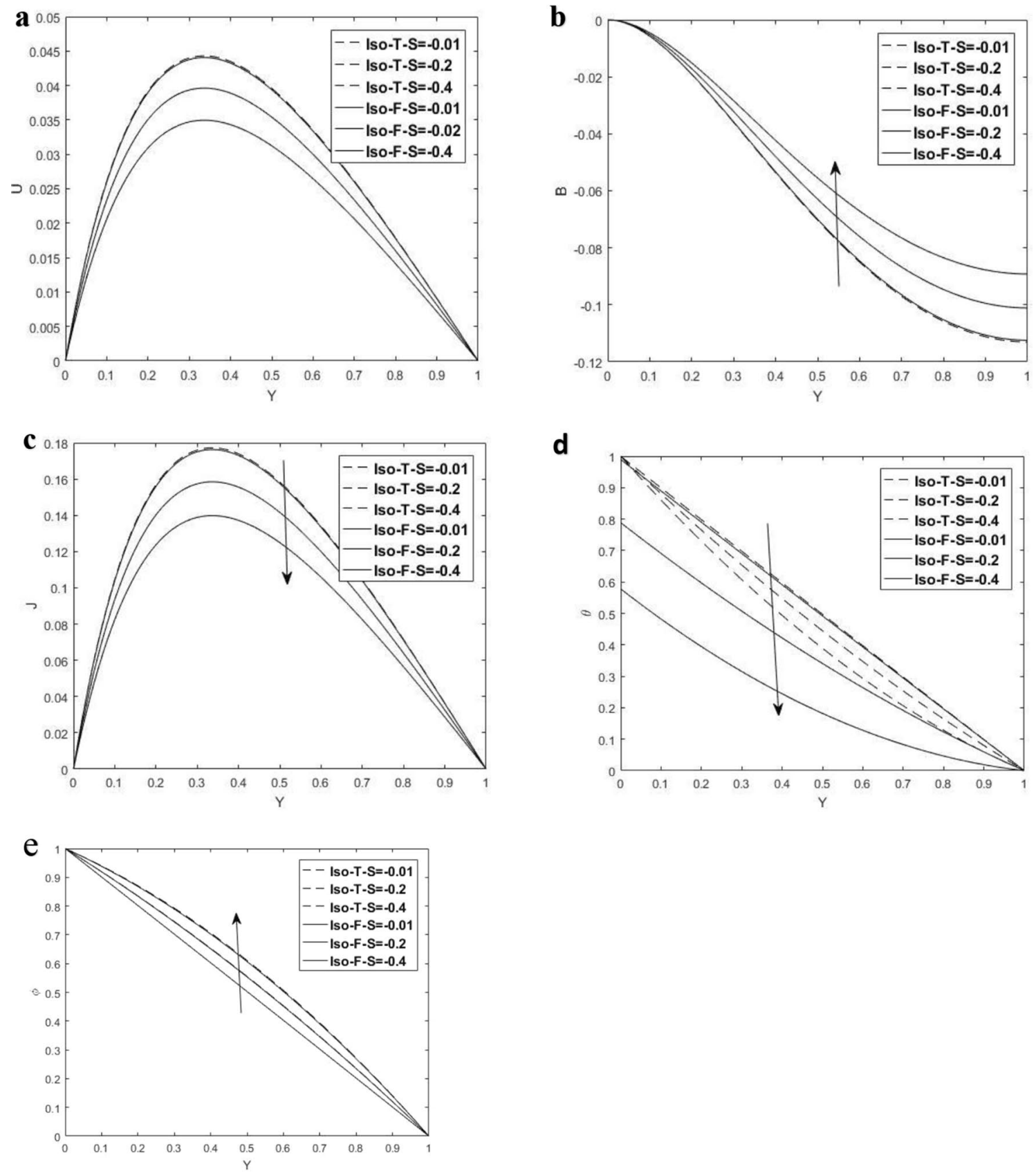

Fig. 8 Variation of $-S$ a Velocity b Induced magnetic field c Induced current density d Temperature and e. Concentration with $\mathrm{Nb}=0.1, \mathrm{Nt}=0.1, \mathrm{Ha}=5, \mathrm{Pm}=0.8, \mathrm{Br}=1$ 
Table 2 Effect of heat source/sink on skin friction for isoflux boundary condition $(\mathrm{Pm}=0.5, \mathrm{Br}=1, \mathrm{Nb}=0.1, \mathrm{Ha}=5, \mathrm{Nt}=0.1)$

\begin{tabular}{llll}
\hline$S$ & Skin friction & $S$ & Skin friction \\
\hline 0.01 & 0.244128 & -0.01 & 0.241558 \\
0.1 & 0.255704 & -0.1 & 0.230003 \\
0.3 & 0.281486 & -0.3 & 0.204383 \\
0.5 & 0.307348 & -0.5 & 0.178844 \\
0.8 & 0.346294 & -0.8 & 0.140687 \\
1 & 0.372359 & -1 & 0.115350 \\
1.2 & 0.398505 & -1.2 & 0.090095 \\
1.5 & 0.437876 & -1.5 & 0.052360 \\
\hline
\end{tabular}

\section{Compliance with ethical standards}

Conflict of interest The authors declared that there is no conflict of interest.

\section{Appendix A}

$P_{1}=N t+N b$

$P_{2}=\frac{1}{1-\exp \left(m_{1}\right)}, m_{1}=N b+N t$

$P_{3}=1-P_{2}$

$P_{4}=-\frac{N t m_{1}^{2} P_{2}}{N b}$

$P_{5}=1-\frac{P_{4}}{m_{1}^{2}}$

$P_{6}=-\frac{P_{4} \exp \left(m_{1}\right)}{m_{1}^{2}}-P_{5}$

$P_{7}=P_{3}+B r P_{5}$

$P_{8}=\frac{B r P_{6}}{2}$

$P_{9}=\frac{P_{2}}{m_{1}}+\frac{B r P_{4}}{m_{1}^{3}}$

$P_{10}=\mathrm{HaPmP}_{7}$

$P_{11}=\mathrm{HaPmP}_{8}$
$P_{12}=\mathrm{HaPmP}_{9}$

$P_{13}=H a^{2} P m$

$P_{14}=-\frac{P_{10}}{P_{13}}$

$P_{15}=-\frac{2 P_{11}}{P_{13}^{2}}$

$P_{16}=-\frac{P_{11}}{P_{13}}$

$P_{17}=\frac{P_{12}}{m_{1}^{2}-P_{13}}$

$P_{18}=-\frac{1}{P_{13}}$

$P_{19}=m_{2} \exp \left(m_{2}\right)$,

$m_{2}=-\sqrt{H a^{2} P m}$

$P_{20}=m_{3} \exp \left(m_{3}\right)$,

$m_{3}=\sqrt{H a^{2} P m}$

$P_{21}=P_{14}+2 P_{16}+$

$m_{1} P_{17} \exp \left(m_{1}\right)$

$P_{22}=P_{19}-P_{20}$

$P_{23}=P_{15} P_{19}-P_{21}$

$P_{24}=P_{18} P_{19}$

$P_{25}=P_{20}-P_{19}$

$P_{26}=P_{15} P_{20}-P_{21}$

$P_{27}=P_{18} P_{20}$

$P_{28}=-\frac{P_{23}}{P_{22}}$ 


$$
\begin{aligned}
& P_{29}=-\frac{P_{24}}{P_{22}} \\
& P_{30}=-\frac{P_{26}}{P_{25}} \\
& P_{31}=-\frac{P_{27}}{P_{25}} \\
& P_{32}=-\frac{1}{H a P m} \\
& p_{33}=m_{2} P_{30}+m_{3} P_{28} \\
& P_{34}=m_{2} P_{31}+m_{3} P_{29} \\
& P_{35}=m_{1} P_{17}+P_{14} \\
& P_{36}=P_{33}+P_{35} \\
& P_{37}=P_{32} P_{36} \\
& P_{38}=P_{32} P_{34} \\
& P_{39}=m_{2} P_{30} \exp \left(m_{2}\right)+m_{3} P_{28} \exp \left(m_{3}\right) \\
& P_{40}=m_{2} P_{31} \exp \left(m_{2}\right)+m_{3} P_{29} \exp \left(m_{3}\right) \\
& P_{41}=m_{1} P_{17} \exp \left(m_{1}\right)+2 P_{16}+P_{14} \\
& P_{42}=P_{39}+P_{41} \\
& P_{43}=P_{32} P_{42} \\
& P_{44}=P_{32} P_{40} \\
& P_{45}=P_{37}-P_{43} \\
& P_{46}=P_{38}-P_{44} \\
& P_{47}=-\frac{P_{45}}{P_{46}} \\
& P_{48}=P_{37} P_{44}-P_{43} P_{38} \\
& P_{49}=P_{44}-P_{38}
\end{aligned}
$$$$
P_{50}=-\frac{P_{48}}{P_{49}}
$$$$
P_{51}=P_{30}+P_{47} P_{31}
$$$$
P_{52}=P_{28}+P_{47} P_{29}
$$$$
P_{53}=P_{15}+P_{47} P_{18}
$$$$
P_{54}=P_{50}+P_{14} P_{32}
$$$$
P_{55}=2 P_{32} P_{16}
$$$$
P_{56}=m_{1} P_{32} P_{17}
$$

\section{References}

1. Choi SU, Eastman JA (1995) Enhancing thermal conductivity of fluids with nanoparticles, Argonne National Lab., IL, Rep

2. Ahmad A, Asghar S, Afzal S (2016) Flow of nanofluid past a Riga plate. J Magn Magn Mater 402:44-48

3. Sheremet MA, Oztop HF, Pop I (2016) MHD natural convection in an inclined wavy cavity with corner heater filled with a nanofluid. J Magn Magn Mater 416:37-47

4. Sheikholeslami M, Rokni HB (2017) Nanofluid two phase model analysis in existence of induced magnetic field. Int J Heat Mass Transf 107:288-299

5. Sheikholeslami M, Shehzad SA, Abbasi FM, Li Z (2018) Nanofluid flow and forced convection heat transfer due to Lorentz forces in a porous lid driven cubic enclosure with hot obstacle. Comput Methods Appl Mech Eng 338:491-505

6. Sheikholeslami M (2019) Numerical approach for MHD Al2O3water nanofluid transportation inside a permeable medium using innovative computer method. Comput Methods Appl Mech Eng 344:306-318

7. Vinod S, Philip J (2020) Impact of field ramp rate on magnetic field assisted thermal transport in ferrofluids. J Mol Liq 298:112047

8. Sheikholeslami M, Gorji-Bandpy M, Ganji DD (2014) MHD free convection in an eccentric semi-annulus filled with nanofluid. J Taiwan Inst Chem Eng 45(4):1204-1216

9. Sheikholeslami M, Ashorynejad HR, Rana P (2016) Lattice boltzmann simulation of nanofluid heat transfer enhancement and entropy generation. J Mol Liq 214:86-95

10. Ibrahim W, Makinde OD (2013) The effect of double stratification on boundary-layer flow and heat transfer of nanofluid over a vertical plate. Comput Fluids 86:433-441

11. Sheikholeslami M, Gorji-Bandpy M, Domairry G (2013) Free convection of nanofluid filled enclosure using lattice Boltzmann method (LBM). Appl Math Mech 34(7):833-846 
12. Sheikholeslami M, Gorji-Bandpy M, Ganji DD (2014) Lattice Boltzmann method for MHD natural convection heat transfer using nanofluid. Powder Technol 254:82-93

13. Makinde OD, Aziz A (2011) Boundary layer flow of a nanofluid past a stretching sheet with a convective boundary condition. Int J Therm Sci 50(7):1326-1332

14. Mutuku WN, Makinde OD (2014) Hydromagnetic bioconvection of nanofluid over a permeable vertical plate due to gyrotactic microorganisms. Comput Fluids 95:88-97

15. Hayat T, Waqas M, Khan MI, Alsaedi A (2016) Analysis of thixotropic nanomaterial in a doubly stratified medium considering magnetic field effects. Int J Heat Mass Transf 102:1123-1129

16. Khan WA, Makinde OD (2014) MHD nanofluid bioconvection due to gyrotactic microorganisms over a convectively heat stretching sheet. Int J Therm Sci 81:118-124

17. Makinde OD, Ogulu A (2008) The effect of thermal radiation on the heat and mass transfer flow of a variable viscosity fluid past a vertical porous plate permeated by a transverse magnetic field. Chem Eng Commun 195(12):1575-1584

18. Sarveshanand, Singh AK (2015) Magnetohydrodynamic free convection between vertical parallel porous plates in the presence of induced magnetic field. Springer Plus 4(1):333

19. Cao L, Liu D, Jiang P, Shao X, Zhou Q, Wang Y (2019) Multi-physics simulation of dendritic growth in magnetic field assisted solidification. Int J Heat Mass Transf 144:118673

20. Feng L, Shi W-Y, Shoji E, Kubo M, Tsukada T (2019) Effects of vertical, horizontal and rotational magnetic fields on convection in an electromagnetically levitated droplet. Int J Heat Mass Transf 130:787-796

21. Mehryan SAM, Tahmasebi A, Izadi M, Ghalambaz M (2020) Melting behavior of phase change materials in the presence of a non-uniform magnetic-field due to two variable magnetic sources. Int J Heat Mass Transf 149:119184

22. Lavanya B, Ratnam AL (2014) Radiation and mass transfer effects on unsteady MHD free convective flow past a vertical porous plate embedded in a porous medium in a slip flow regime with heat source/sink and soret effect. Int J Eng Tech Res 2(11):210-220

23. Kumar D, Singh AK (2016) Effects of heat source/sink and induced magnetic field on natural convective flow in vertical concentric annuli. Alexandria Eng J 55(4):3125-3133

24. Alsabery Al, Gedik E, Chamkha AJ, Hashim I (2019) Effects of two-phase nanofluid model and localized heat source/sink on natural convection in a square cavity with a solid circular cylinder. Comput Methods Appl Mech Eng 346:952-981

25. Tlau L, Ontela S (2019) Entropy generation in MHD nanofluid flow with heat source/sink. SN Appl Sci 1(12):1672

26. Chu W-X, Tsai M-K, Jan S-Y, Huang H-H, Wang C-C (2020) CFD analysis and experimental verification on a new type of aircooled heat sink for reducing maximum junction temperature. Int J Heat Mass Transf 148:119094

27. Wang X, Chen M, Tate D, Rahimi H, Zhang S (2020) Numerical investigation on hydraulic and thermal characteristics of micro latticed pin fin in the heat sink. Int J Heat Mass Transf 149:119157

28. Roy NC, Hossain MA, Gorla RSR (2020) Natural convection in a cavity with trapezoidal heat sources mounted on a square cylinder. SN Appl Sci 2(2):1-11

Publisher's Note Springer Nature remains neutral with regard to jurisdictional claims in published maps and institutional affiliations. 